\title{
BOUNDARY VALUE PROBLEM FOR A TWO-TIME-SCALE NONLINEAR DISCRETE SYSTEM
}

\author{
Tahia Zerizer \\ Mathematics Department \\ College of Sciences \\ Jazan University, Jazan, Kingdom of SAUDI ARABIA
}

\begin{abstract}
In this work, an algorithmic procedure is given to implement the solution of a two-point boundary value problem for a nonlinear two-time-scale discrete-time system.
\end{abstract}

AMS Subject Classification: 93C05, 93C55, 93C70, 93C73

Key Words: discrete system, time-scale, iterative methods, boundary value problem, asymptotic expansions

\section{Introduction}

Singularly perturbed discrete systems (SPDSs) occur in engineering and applied mathematics. Contrary to the continuous-time counterpart, they could be characterized by numerous models (see [3], [12], [13], [14], [15], [16]). The details on the recent progress of the theory and applications of SPDSs can be found from the survey paper [3] and the references therein. In this article we study a class of nonlinear SPDS introduced in [6] and [9],

$$
\left\{\begin{array}{l}
x(t+1)=\varepsilon f(x(t), y(t), \varepsilon, t), \quad t \in I_{N-1}, \\
y(t+1)=g(x(t), y(t), \varepsilon, t),
\end{array}\right.
$$

where $\varepsilon$ is a small real parameter, and $I_{N}=\{0,1, \cdots, N\}, N$ a positive integer. Let $F\left(I_{N}, X\right)$ and $G\left(I_{N}, Y\right)$ denote the space of all mappings of $I_{N}$ into the Banach spaces $(X,\|\cdot\|)$ and $(Y,\|\cdot\|)$, respectively, $\mathcal{U}:=F\left(I_{N}, X\right) \times G\left(I_{N}, Y\right) \times$

Received: December 5, 2018

(C) 2019 Academic Publications 
$(-1,1) \times I_{N}$; the mappings $f: \mathcal{U} \rightarrow X$ and $g: \mathcal{U} \rightarrow Y$ are supposed to be $n$-differentiable in their arguments. In the next section, we associate to (1) two known boundary conditions

$$
x(t=0)=\alpha(\varepsilon), \quad y(t=N)=\beta(\varepsilon) .
$$

For sufficiently small values of the perturbation parameter $\varepsilon$, we study the existence and uniqueness of a solution $(x(t, \varepsilon), y(t, \varepsilon)), t \in I_{N}$, and we seek its asymptotic expansion

$$
\begin{aligned}
& x(t, \varepsilon)=x(t)^{(0)}+\varepsilon x(t)^{(1)}+\cdots+\varepsilon^{n} x(t)^{(n)}+\mathcal{O}\left(\varepsilon^{n+1}\right), \\
& y(t, \varepsilon)=y(t)^{(0)}+\varepsilon y(t)^{(1)}+\cdots+\varepsilon^{n} y(t)^{(n)}+\mathcal{O}\left(\varepsilon^{n+1}\right),
\end{aligned}
$$

by using an algorithmic technique we developed firstly for perturbed difference equations (see [10], [11], [16], [17], [18], [19]). Recently, many researchers have studied discrete versions of boundary value problems (BVPs) (see [1], [4], [5], [8]), and applications of two-point BVP algorithms arise in pollution control problems, nuclear reactor heat transfer and vibration. For an abbreviated writing, we denote the partial derivative $\frac{\partial^{k_{1}+k_{2}+\cdots+k_{p}} f\left(x_{1}, x_{2}, \cdots, x_{p}\right)}{\partial x_{1}^{k_{1}} \partial x_{2}^{k_{2} \ldots \partial x_{p}^{k_{p}}}}$ by $D_{1}^{k_{1}} D_{2}^{k_{2}} \cdots D_{p}^{k_{p}} f$.

\section{Main Result}

This section contains the main results of the article, we describe the algorithmic procedure providing the asymptotic solutions. For $|\varepsilon|<\delta \leq 1$, we assume that the boundary values $\alpha(\varepsilon)$ and $\beta(\varepsilon)$, have the asymptotic representations

$$
\begin{aligned}
& \alpha(\varepsilon)=\alpha^{(0)}+\varepsilon \alpha^{(1)}+\cdots+\varepsilon^{n} \alpha^{(n)}+\mathcal{O}\left(\varepsilon^{n+1}\right), \\
& \beta(\varepsilon)=\beta^{(0)}+\varepsilon \beta^{(1)}+\cdots+\varepsilon^{n} \beta^{(n)}+\mathcal{O}\left(\varepsilon^{n+1}\right) .
\end{aligned}
$$

\subsection{Reduced Problem}

By setting the small parameter to zero in $(5)-(6)$, results the lower order subsystem, said reduced problem

$$
\begin{gathered}
\left\{\begin{array}{l}
x^{(0)}(t+1)=0 \\
y^{(0)}(t+1)=g\left(x^{(0)}(t), y^{(0)}(t), 0, t\right), \quad t \in I_{N-1},
\end{array}\right. \\
x^{(0)}(0)=\alpha(0), \quad y^{(0)}(N)=\beta(0) .
\end{gathered}
$$


It is seen that the states $x^{(0)}(t), 1 \leq t \leq N$, are fixed

$$
x^{(0)}(0)=\alpha(0), \quad x^{(0)}(1)=x^{(0)}(2)=\cdots=x^{(0)}(N)=0,
$$

whereas the states $y^{(0)}(t), 0 \leq t \leq N$, satisfy the recurrence

$$
\left\{\begin{array}{l}
y^{(0)}(1)=g\left(\alpha(0), y^{(0)}(t), 0,0\right), \\
y^{(0)}(t+1)=g\left(0, y^{(0)}(t), 0, t\right), 1 \leq t \leq N-1, y^{(0)}(N)=\beta(0),
\end{array}\right.
$$

which needs only the final value to be solved backwards, thus the boundary layer occurs at the initial value $x^{(0)}(0)=\alpha(0)$. To resolve BVP $(5)-(6)$, we assume the following hypothesis.

H1 $D_{2} g(x(t), y(t), 0, t) \neq 0, \quad \forall(x, y) \in X \times Y, \quad t=0, \ldots, N-1$.

Proposition 1. If $H 1$ holds, then BVP (5)-(6) has a unique solution.

\subsection{Preliminaries}

We use the notation

$$
\mathcal{X}=(x(0), y(0), x(1), y(1), \ldots, x(N), y(N)),
$$

thus system $(1)-(2)$ can be written in the form $\mathcal{F}(\varepsilon, \mathcal{X})=0$, where

$$
\begin{gathered}
\mathcal{F}:(-1,1) \times X^{2 N+2} \longrightarrow X^{2 N+2}, \\
\mathcal{F}(\varepsilon, \mathcal{X})=\left(f_{0}(\varepsilon, \mathcal{X}), g_{0}(\varepsilon, \mathcal{X}), \ldots, f_{N}(\varepsilon, \mathcal{X}), g_{N}(\varepsilon, \mathcal{X})\right) \\
\left\{\begin{array}{l}
f_{0}(\varepsilon, \mathcal{X})=x(0)-\alpha(\varepsilon), \\
f_{t}(\varepsilon, \mathcal{X})=x(t+1)-\varepsilon f(x(t), y(t), \varepsilon, t), \quad t=0, \cdots, N-1 . \\
g_{t}(\varepsilon, \mathcal{X})=y(t+1)-g(x(t), y(t), \varepsilon, t), \\
g_{N}(\varepsilon, \mathcal{X})=y(N)-\beta(\varepsilon),
\end{array}\right.
\end{gathered}
$$

For a sufficiently small parameter $\varepsilon$, with Hypothesis $\mathrm{H} 1$ and other appropriate assumptions, the classical Implicit Function Theorem [2] assures the existence of a function

$$
\Gamma(\varepsilon)=\left(\phi_{0}(\varepsilon), \psi_{0}(\varepsilon), \phi_{1}(\varepsilon), \psi_{1}(\varepsilon), \ldots, \phi_{N}(\varepsilon), \psi_{N}(\varepsilon)\right),
$$

of class $C^{n}$, such that $\mathcal{F}(\varepsilon, \Gamma(\varepsilon))=0$. Therefore, we have

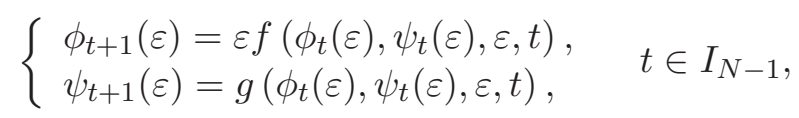




$$
\phi_{0}(\varepsilon)=\alpha(\varepsilon), \quad \psi_{N}(\varepsilon),=\beta(\varepsilon) .
$$

We aim to determine the coefficients of the polynomial expansions (Taylor/Maclaurin)

$$
\begin{aligned}
& \phi_{t}(\varepsilon)=\phi_{t}(0)+\frac{\varepsilon}{1 !} \frac{d \phi_{t}}{d \varepsilon}(0)+\frac{\varepsilon^{2}}{2 !} \frac{d^{2} \phi_{t}}{d \varepsilon^{2}}(0)+\cdots+\frac{\varepsilon^{n}}{n !} \frac{d^{n} \phi_{t}}{d \varepsilon^{n}}(0)+\mathcal{O}\left(\varepsilon^{n+1}\right), \\
& \psi_{t}(\varepsilon)=\psi_{t}(0)+\frac{\varepsilon}{1 !} \frac{d \psi_{t}}{d \varepsilon}(0)+\frac{\varepsilon^{2}}{2 !} \frac{d^{2} \psi_{t}}{d \varepsilon^{2}}(0)+\cdots+\frac{\varepsilon^{n}}{n !} \frac{d^{n} \psi_{t}}{d \varepsilon^{n}}(0)+\mathcal{O}\left(\varepsilon^{n+1}\right)
\end{aligned}
$$

therefore, we have to find explicitly the sequential differentiation of (10). It is achieved in the following Lemma by using the formula of Faa di Bruno [7].

Lemma 2. Suppose that $\phi$ and $\psi$ satisfy (10), and that all necessary derivatives are defined. Then we have for $n \geq 2$,

$$
\begin{aligned}
& \frac{d^{n} \phi_{t+1}}{d \varepsilon^{n}}(0)=\sum_{0} \sum_{1} \cdots \sum_{n-1} \frac{n ! D_{1}^{p_{1}} D_{2}^{p_{2}} D_{3}^{p_{3}} f_{t} \prod_{i=1}^{n-1}\left(\frac{d^{i} \phi_{t}(0)}{d \varepsilon^{i}}\right)^{q_{i 1}\left(\frac{d^{i} \psi_{t}(0)}{d \varepsilon^{i}}\right)^{q_{i 2}} \delta_{i}}}{\prod_{i=1}^{n-1}(i !)^{k_{i}} \prod_{i=1}^{n-1} \prod_{j=1}^{3} q_{i j} !} \\
& \frac{d^{n} \psi_{t+1}}{d \varepsilon^{n}}(0)=\sum_{0} \sum_{1} \cdots \sum_{n} \frac{n ! D_{1}^{p_{1}} D_{2}^{p_{2}} D_{3}^{p_{3}} g_{t} \times \prod_{i=1}^{n}\left(\frac{d^{i} \phi_{t}(0)}{d \varepsilon^{i}}\right)^{q_{i 1}\left(\frac{d^{i} \psi_{t}(0)}{d \varepsilon^{i}}\right)^{q_{i}} 2 \delta_{i}}}{\prod_{i=1}^{n}(i !)^{k_{i}} \prod_{i=1}^{n} \prod_{j=1}^{3} q_{i j} !},
\end{aligned}
$$

where the coefficients $k_{i}, q_{i j}$ and $p_{j}, i=0, \cdots, n, j=1,2,3$, are all nonnegative integer solutions of the Diophantine equations

$$
\begin{gathered}
\sum_{0} \rightarrow k_{1}+2 k_{2}+\cdots+n k_{n}=n, \\
\sum_{i} \rightarrow q_{i 1}+q_{i 2}+q_{i 3}=k_{i}, \quad i=1,2, \cdots, n, \\
p_{j}=q_{1 j}+q_{2 j}+\cdots+q_{n j}, \quad j=1,2,3, \\
k=p_{1}+p_{2}+p_{3}=k_{1}+k_{2}+\cdots+k_{n},
\end{gathered}
$$

and

$$
\begin{gathered}
f_{t}:=f\left(\phi_{t}(0), \psi_{t}(0), 0, t\right), \quad g_{t}:=g\left(\phi_{t}(0), \psi_{t}(0), 0, t\right), \\
\delta_{i}:=\left(\varepsilon^{(i)}\right)^{q_{i 3}}=\left\{\begin{array}{llll}
1, & i=1 & \vee & q_{i 3}=0, \\
0, & i \geq 2 & \wedge & q_{i 3} \neq 0 .
\end{array}\right.
\end{gathered}
$$

Proof. We expand Faa Di Bruno's Formula into (10), and we verify by induction

$$
\left.\frac{d^{n}}{d \varepsilon^{n}}\left(\varepsilon f\left(\phi_{t}(\varepsilon), \psi_{t}(\varepsilon), \varepsilon, t\right)\right)\right|_{\varepsilon=0}=\left.n \frac{d^{n-1}}{d \varepsilon^{n-1}}\left(f\left(\phi_{t}(\varepsilon), \psi_{t}(\varepsilon), \varepsilon, t\right)\right)\right|_{\varepsilon=0}
$$




\subsection{Description of the Method}

We find an algorithmic process giving the coefficients of (3) when we substitute, into (13) and (14), for $0 \leq t \leq N, l=0,1$, by

$$
x^{(i)}(t+l):=\frac{1}{i !} \frac{d^{i} \phi_{t+l}}{d \varepsilon^{i}}(0), y^{(i)}(t+l):=\frac{1}{i !} \frac{d^{i} \psi_{t+l}}{d \varepsilon^{i}}(0) .
$$

Zero order approximation coefficients refer to the sequence solution of the reduced problem $(5)-(6)$, thus $x^{(0)}(t)$ verify $(7)$ and hypothesis H1 allows computing $y^{(0)}(t)$ from $(8)$. For first order coefficients, we obtain the forward recurrence

$$
\begin{aligned}
& x^{(1)}(0)=\alpha^{(1)}, \quad x^{(1)}(1)=f\left(\alpha(0), y^{(0)}(0), 0,0\right), \\
& x^{(1)}(t+1)=f\left(0, y^{(0)}(t), 0, t\right), 1 \leq t \leq N,
\end{aligned}
$$

and the difference equation

$$
\begin{aligned}
& y^{(1)}(t)=\left[D_{2} g_{t}^{(0)}\right]^{-1}\left[y^{(1)}(t+1)-D_{1} g_{t}^{(0)} x^{(1)}(t)-D_{3} g_{t}^{(0)}\right] \\
& y^{(1)}(N)=\beta^{(1)}
\end{aligned}
$$

which may be solved backwards with the final value. For second order development, we determine $x^{(0)}(t)$ by straightforward computation from the difference equation

$$
\begin{gathered}
x^{(2)}(0)=\alpha^{(2)}, \\
x^{(2)}(t+1)=D_{1} f_{t}^{(0)} x^{(1)}(t)+D_{2} f_{t}^{(0)} y^{(1)}(t)+D_{3} f_{t}^{(0)}, t \in I_{N-1},
\end{gathered}
$$

then we can calculate $y^{(0)}(t)$ backwards from the recurrence

$$
\begin{gathered}
y^{(2)}(t)=\left[D_{2} g_{t}^{(0)}\right]^{-1}\left[y^{(2)}(t+1)-D_{1} g_{t}^{(0)} x^{(2)}(t)-\frac{1}{2 !} D_{3}^{2} g_{t}^{(0)}\right. \\
-\frac{1}{2 !} D_{1}^{2} g_{t}^{(0)}\left(x^{(1)}(t)\right)^{2}-\frac{1}{2 !} D_{2}^{2} g_{t}^{(0)}\left(y^{(1)}(t)\right)^{2}-D_{1} D_{3} g_{t}^{(0)} x^{(1)}(t) \\
\left.-D_{2} D_{3} g_{t}^{(0)} y^{(1)}(t)-D_{1} D_{2} g_{t}^{(0)} x^{(1)}(t) y^{(1)}(t)\right] \\
y^{(2)}(N)=\beta^{(2)} .
\end{gathered}
$$

In general, to find the $n$ order coefficients, first we perform the forward iteration

$$
\begin{gathered}
x^{(n)}(0)=\alpha^{(n)} \\
x^{(n)}(t+1)=\sum_{0} \cdots \sum_{n-1} \frac{D_{1}^{p_{1}} D_{2}^{p_{2}} D_{3}^{p_{3}} f_{t}^{(0)} \prod_{i=1}^{n-1}\left(x^{(i)}(t)\right)^{q_{i 1}\left(y^{(i)}(t)\right)^{q_{i 2}} \delta_{i}}}{\prod_{i=1}^{n-1} \prod_{j=1}^{3} q_{i j} !}
\end{gathered}
$$


then after we solve backwards the difference equation

$$
\begin{aligned}
& y^{(n)}(t)=\left[D_{2} g_{t}^{(0)}\right]^{-1}\left[y^{(n)}(t+1)-D_{1} g_{t} x^{(n)}(t)\right.
\end{aligned}
$$

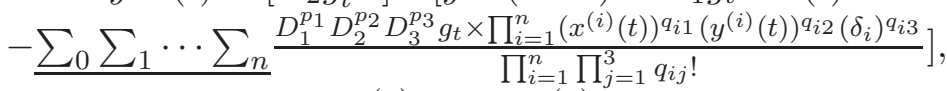

$$
\begin{aligned}
& y^{(n)}(N)=\beta^{(n)} .
\end{aligned}
$$

The following theorem illustrates the effectiveness of the suggested algorithm.

Theorem 3. If $H 1$ holds, there exists $\epsilon>0$, such that for all $|\varepsilon|<\epsilon$, the boundary value problem (1) - (2) has a unique solution which satisfies (3); the coefficients $x^{(0)}(t), y^{(0)}(t), x^{(1)}(t), y^{(1)}(t), x^{(2)}(t), y^{(2)}(t), x^{(n)}(t), y^{(n)}(t)$, are found following the ordered iterative process (7), (8), (20), (21), (22), (23), (24), (25), respectively.

Proof. Let $\mathrm{F}(\tilde{\mathcal{X}})=(\varepsilon, \mathcal{F}(\tilde{\mathcal{X}}))$ where $\mathcal{D F}$ denotes its jacobian matrix, $\tilde{\mathcal{X}}=(\varepsilon, \mathcal{X}),|\varepsilon| \leq \delta<1$. We have from hypothesis $\mathrm{H} 1$

$$
\operatorname{det} \mathcal{D F}\left(\tilde{\mathcal{X}}^{(0)}\right)=\prod_{t=0}^{N-1} \mathcal{D}_{2} g\left(x^{(0)}(t), y^{(0)}(t), 0, t\right) \neq 0
$$

which justifies that at

$$
\tilde{\mathcal{X}}^{(0)}=\left(0, x^{(0)}(0), y^{(0)}(0), x^{(0)}(1), y^{(0)}(1), \ldots, x^{(0)}(N), y^{(0)}(N)\right),
$$

the inverse of $\mathcal{D F}$ exists and (5)-(6) has a unique solution. We can choose $\xi>0$ such that, if $\left\|\tilde{\mathcal{X}}-\tilde{\mathcal{X}}^{(0)}\right\|<\xi$, we have

$$
\left\|\mathcal{D F}(\tilde{\mathcal{X}})-\mathcal{D F}\left(\tilde{\mathcal{X}}^{(0)}\right)\right\|<\frac{1}{2}\left\|\left(\mathcal{D F}\left(\tilde{\mathcal{X}}^{(0)}\right)\right)^{-1}\right\|^{-1},
$$

since $\mathcal{D F}$ is continuous. Let $\epsilon=\frac{\xi}{2}\left\|\left(\mathcal{D F}\left(\tilde{\mathcal{X}}^{(0)}\right)\right)^{-1}\right\|^{-1}$, the mapping

$$
\Omega_{\tau}(\tilde{\mathcal{X}})=\tilde{\mathcal{X}}-\left(\mathcal{D F}\left(\tilde{\mathcal{X}}^{(0)}\right)\right)^{-1}(\mathrm{~F}(\tilde{\mathcal{X}})-\tau)
$$

is a contraction from $B\left(\tilde{\mathcal{X}}^{(0)}, \xi\right)$ to itself, when $|\varepsilon|<\epsilon$ and $\|\tau\|<\epsilon$. Then $\Omega_{\tau}$ has a unique fixed point $\tilde{\mathcal{X}}$. Therefore, for $\tau$ fixed, $\|\tau\|<\epsilon$, there exists a unique $\tilde{\mathcal{X}}$ such that $\left\|\tilde{\mathcal{X}}-\tilde{\mathcal{X}}^{(0)}\right\|<\xi$, and $\tau=\mathrm{F}(\tilde{\mathcal{X}})$, i.e., $\mathrm{F}$ is 1-to1 from $\mathrm{F}^{-1}(B(0, \epsilon))$ into $B(0, \epsilon)$. Suppose that $|\varepsilon|<\epsilon$, therefore, we have 
$(\varepsilon, 0, \cdots, 0) \in B(0, \epsilon)$, there exists a unique $(\varepsilon, \Gamma(\varepsilon))$ in $B\left(\tilde{\mathcal{X}}^{(0)}, \xi\right)$, such that $(\varepsilon, 0, \cdots, 0)=\mathrm{F}(\varepsilon, \Gamma(\varepsilon))$, where $\Gamma(\varepsilon)=\left(\phi_{0}(\varepsilon), \psi_{0}(\varepsilon), \cdots, \phi_{N}(\varepsilon), \psi_{N}(\varepsilon)\right)$. We proved that $|\varepsilon|<\epsilon$, there exists a unique $\phi(\varepsilon)$ such that $\mathcal{F}(\varepsilon, \Gamma(\varepsilon))=0$, then $\operatorname{BVP}(1)-(2)$ has a unique solution. In addition, as are $\mathrm{F}$ and $\mathrm{F}^{-1}$, the function $\Gamma$ is $C^{n}(-\epsilon, \epsilon)$, and Lemma 2 gives its derivatives.

Iterative problems given above are defined for all order if $f$ and $g$ are smooth functions and the asymptotic developments for the boundary conditions are convergent.

H2 Assume that $\left\|\alpha_{k}^{(i)}\right\| \leq \frac{A}{\delta^{i}},\left\|\beta^{(i)}\right\| \leq \frac{B}{\delta^{i}}, A$ and $B$ are constants.

Theorem 4. If assumptions $\mathrm{H} 1$ and $\mathrm{H} 2$ hold, and $f$ is a smooth function, then there exists $\epsilon>0$, for all $|\varepsilon|<\epsilon$, the boundary value problem (1)-(2) has a unique solution which satisfies

$$
\left.x(t, \varepsilon)=\sum_{n=0}^{\infty} \varepsilon^{n} x^{(n)}(t), \quad y(t, \varepsilon)\right)=\sum_{n=0}^{\infty} \varepsilon^{n} y^{(n)}(t),
$$

where $x^{(0)}(t), y^{(0)}(t), x^{(1)}(t), y^{(1)}(t), x^{(2)}(t), y^{(2)}(t), x^{(n)}(t), y^{(n)}(t)$, are the solutions of the problems (7), (8), (20), (21), (22), (23), (24), (25), respectively.

\section{Conclusion}

We have addressed a two-time-scale nonlinear discrete system. A BVP has been analyzed using the perturbation method and an iterative algorithm is given to find asymptotic solutions at any order. The same technique can be helpful for the IVP since it has the advantage to suppress time scales generating error accumulation, and provides reliable results with few iterations.

\section{References}

[1] A. Golev, S. Hristova, S. Nenov, Monotone-iterative method for solving antiperiodic nonlinear boundary value problems for generalized delay difference equations with maxima, Abstr. Appl. Anal., 2013 (2013), Article ID 571954 . 
[2] S.G. Krantz, H.R. Parks, The Implicit Function Theorem, History, Theory, and Applications, Birkhäuser, Basel (2003).

[3] G.A. Kurina, M.G. Dmitriev, D.S. Naidu, Discrete singularly perturbed control problems (A Survey), Dyn. Contin. Discrete Impuls. Syst. Ser. B Appl. Algorithms., 24 (2017), 335-370.

[4] A.N. Kvitko, A method for solving boundary value problems for nonlinear control systems in the class of discrete controls, Differentsialnye Uravneniya, 44, No 11 (2008), 1499-1509.

[5] A.N. Kvitko, O.S. Firyulina, A.S. Eremin, Solving boundary value problem for a nonlinear stationary controllable system with synthesizing control, Math. Probl. Eng., 2017 (2017), Article ID 8529760.

[6] T.H.S. Li, J.S. Chiou, and F.C. Kung, Stability bounds of singularly perturbed discrete systems, IEEE Trans. Autom. Control, 44, No 10 (1999), 1934-1938.

[7] R.L. Mishkov, Generalization of the formula of Faa Di Bruno for a composite function with a vector argument, Int. J. Math. Math. Sci., 24 (2000), 481-491.

[8] P.Y.H. Pang, R.P. Agarwal, Monotone iterative methods for a general class of discrete boundary value problems, Computers Math. Applic., 28 (1994), 243-254.

[9] K.S. Park, J.T. Lim, Analysis of nonstandard nonlinear singularly perturbed discrete systems, IEEE Trans Circuits Syst-II: Express Briefs, 58, No 5 (2011).

[10] T. Sari, T. Zerizer, Perturbations for linear difference equations, J. Math. Anal. Appl., 1 (2005), 43-52.

[11] T. Zerizer, Perturbation method for linear difference equations with small parameters, Adv. Differ. Equ., 11 (2006), 1-12, Article ID 19214.

[12] T. Zerizer, Perturbation method for a class of singularly perturbed systems, Adv. Dyn. Syst. Appl., 9, No 2 (2014), 239-248.

[13] T. Zerizer, Problems for a linear two-time-scale discrete model, Adv. Dyn. Syst. Appl., 10, No 1 (2015), 85-96. 
[14] T. Zerizer, Boundary value problems for linear singularly perturbed discrete systems, Adv. Dyn. Syst. Appl., 10, No 2 (2015), 215-224.

[15] T. Zerizer, Boundary value problem for a three-time-scale singularly perturbed discrete system, Dyn. Contin. Discrete Impuls. Syst. Ser. A Math. Anal., 23 (2016), 263-272.

[16] T. Zerizer, On a class of perturbed discrete nonlinear systems, GJPAM, 14, No 10 (2018), 1407-1418.

[17] T. Zerizer, A class of nonlinear perturbed difference equations, Int. J. Math. Anal., 12, No 5 (2018), 235-243.

[18] T. Zerizer, A class of multi-scales nonlinear difference equations, Appl. Math. Sci., 12, No 19 (2018), 911-919.

[19] T. Zerizer, Nonlinear perturbed difference equations, J. Nonlinear Sci. Appl., 11 (2018), 1355-1362. 
